\title{
Florid Vascular Proliferation of the Colon Related to Intussusception and Mucosal Prolapse: Potential Diagnostic Confusion with Angiosarcoma
}

Neil R. Bavikatty, M.D., John R. Goldblum, M.D., Fadi W. Abdul-Karim, M.D., Surl L. Nielsen, M.D., Joel K. Greenson, M.D.

Department of Pathology (NRB, JKG), University of Michigan Hospitals, Ann Arbor, Michigan; Department of Anatomic Pathology (JRG), The Cleveland Clinic Foundation, Cleveland, Ohio; Department of Pathology (FWA), University Hospitals of Cleveland, Cleveland, Ohio; and Department of Pathology (SLN), Sutter General Hospital, Sacramento, California

With the exception of angiodysplasia, vascular abnormalities of the intestines are unusual. We describe a florid benign vascular proliferation of the colon in five adult patients, three of whom presented with idiopathic intussusception. In all cases, the proliferation was sufficiently exuberant to raise the possibility of angiosarcoma as a diagnostic consideration. The group included 2 males and 3 females with a median age of 43 years. Two patients were HIV positive. Four patients presented with a colonic mass; other symptoms at presentation included abdominal pain, diarrhea, bleeding, and bowel obstruction. In all cases, a florid lobular proliferation of small vascular channels lined by plump endothelial cells extended from the submucosa through the entire thickness of the bowel wall. The endothelial cells showed minimal nuclear atypia, and mitotic figures were infrequent. The overlying mucosa showed ulceration with ischemic-type changes, and had features of mucosal prolapse. A possible underlying arteriovenous malformation was identified in two cases. All patients were alive and well at last follow-up (interval, 6 months to 5 years). The presence of intussusception or mucosal prolapse in all of the cases suggests repeated mechanical forces applied to the bowel wall as a possible etiologic factor. The role of HIV infection in the pathogenesis of these lesions remains to be determined.

Copyright (C) 2001 by The United States and Canadian Academy of Pathology, Inc.

VOL. 14, NO. 11, P. 1114, 2001 Printed in the U.S.A

Date of acceptance: June 6, 2001.

Address reprint requests to: Joel Greenson, M.D., Department of Pathol ogy, 2G332 University Hospital, 1500 E. Medical Center Dr., Ann Arbor, MI 48109-0054; e-mail: facjkgmd@umich.edu; fax: 734-763-4095.
KEY WORDS: Blood vessel, Colon, Human immunodeficiency virus, Intussusception, Mucosal prolapse, Vascular proliferation, Vascular tumor.

Mod Pathol 2001;14(11):1114-1118

With the exception of angiodysplasia, vascular abnormalities of the intestines are unusual. Benign intestinal vascular lesions include telangiectasias, arteriovenous malformations, and hemangiomas (capillary and cavernous). Kaposi's sarcoma of the gastrointestinal tract occurs commonly in patients with the acquired immunodeficiency syndrome (1), whereas angiosarcoma arising in the intestines is extraordinarily rare (2-4).

Ramsden et al. reported a case of a florid vascular proliferation occurring in association with an ileocecal intussusception (5). The proliferation was sufficiently florid to mimic a primary angiomatous lesion. In this report, we describe a similar florid vascular proliferation of the colon in five adult patients, three of whom presented with idiopathic intussusception. In each case, the proliferation was sufficiently exuberant to raise the possibility of angiosarcoma. Based on the histologic and clinical features, we believe this process to be of a reactive rather than neoplastic nature.

\section{METHODS}

The five cases were collected from the files of the University of Michigan Department of Pathology (Cases 1 and 5), the Cleveland Clinic Foundation (Cases 2 and 3), and the University Hospitals of Cleveland (Case 4). Cases 1, 2, and 5 were seen in consultation by one of the authors (JKG, Cases 1 and 5; JRG, Case 2). Immunohistochemical staining for CD31 was performed on a Ventana 320 Automated Immunostainer (Ventana, Tucson, AZ) by 
the avidin-biotin-peroxidase technique using the monoclonal antibody JC70 (DAKO; Carpinteria, $\mathrm{CA})$.

\section{RESULTS}

The group of patients included 2 males and 3 females with a median age of 43 years (range, 36-82 y). Two of the patients were seropositive for the human immunodeficiency virus (HIV). The clinical characteristics of the patients are summarized in Table 1 . All patients were treated by surgical resection. The follow-up interval was 6 months to 5 years (median, 6 months; mean, 23 months).

Histologic examination of each of the colonic resection specimens revealed a florid proliferation of small vascular channels arising adjacent to a chronic ulcer (Fig. 1A). The proliferation extended from the submucosa to the subserosal connective tissue. In four cases (Cases 1, 2, 4, and 5) the vessels extended into the mucosa. At low power, the lesions had a distinctly lobular architecture, similar to a pyogenic granuloma. This lobularity was accentuated on sections immunostained for CD31 (Fig. 1B). The vessels were lined by plump endothelial cells that showed strong expression of CD31 (Fig. 1, C-D). The lesions showed focal areas of increased cellularity with spindled cells (Fig. 2); however, the overall appearance was inconsistent with Kaposi's sarcoma. The endothelial cells lacked significant hyperchromasia, and nuclear pleomorphism was slight. Mitotic figures were encountered infrequently; no atypical mitoses were observed.

Muscular fibroplasia was noted in the lamina propria adjacent to the lesions, suggesting mucosal prolapse. In two of the cases (Cases 1 and 5), ischemic-type changes were present in the surrounding mucosa, including regenerative epithelium with small, atrophic crypts and hyalinization of the lamina propria (Fig. 3). A possible arteriovenous malformation (Fig. 4) was identified in two cases (Cases 3 and 5). There were no changes to suggest chronic inflammatory bowel disease, and no infectious agents were identified. A WarthinStarry stain performed on Case 4 revealed no organisms, excluding bacillary angiomatosis.

\section{DISCUSSION}

We describe five cases of an unusual florid vascular proliferation of the colon in five adult patients. Although the etiology of the lesions is obscure, three of the patients had a documented intussusception, and all cases showed histologic evidence of mucosal prolapse. In each case, the vascular proliferation was associated with a nearby chronic ulcer. In two cases, the surrounding mucosa showed ischemic-type changes, which are known to occur in long-standing intussuseption. These findings, in conjunction with the benign nuclear features of the endothelial cells and lobularity of the lesions, suggest a reactive process. Further support for an indolent process comes from the lack of recurrence in any of the patients after resection, although the follow-up interval was relatively short in three cases.

Intussusception in children is usually idiopathic. In contrast, adult intussusception can usually be attributed to a specific cause $(6-10)$. This tenet has recently been called into question, however, by a study in which many adult cases diagnosed using CT or magnetic resonance imaging were found to be idiopathic (11). The most common etiology of adult intussusception is the presence of a neoplasm. Other reported causes include Meckel's diverticulum, adhesions, lymphoid hyperplasia, and ectopic pancreas, among others (6-10).

There are several reports of intussusception occurring in association with vascular lesions (12-16). Most of these lesions have had the typical histologic appearance of a hemangioma. We found one report of an intussuscepting angiosarcoma (3); in contrast to our cases, this lesion was metastatic at the time of presentation. Ramsden et al. described a florid vascular proliferation mimicking a primary angiomatous lesion in the cecum of a patient with an ileocolic intussusception (5). The lesion consisted of closely packed, thin-walled vascular channels within the mucosa and submucosa occurring in the vicinity of a polypoid submucosal lipoma acting as a lead point to an intussusception. The mucosa adjacent to the polyp showed areas of ulceration and epithelial regeneration. In contrast to this case, our cases showed no additional mass lesion to serve as a lead point. Yao et al. reported three cases of

TABLE 1. Clinical Characteristics of Five Patients with Florid Vascular Proliferations of the Colon

\begin{tabular}{cclclc}
\hline Case & Age (y)/Sex & \multicolumn{1}{c}{ Site } & Intussusception & \multicolumn{1}{c}{ Clinical Features } & Follow-Up/Interval \\
\hline 1 & $82 / \mathrm{F}$ & Tranverse colon & No & Abdominal pain, bloody diarrhea, colonic mass & No recurrence, 5 y \\
2 & $40 / \mathrm{F}$ & Cecum & Yes & Abdominal pain, cecal mass & No recurrence, 6 mo \\
3 & $58 / \mathrm{M}$ & Cecum & Yes & Cecal mass & No recurrence, 6 mo \\
4 & $43 / \mathrm{F}$ & Cecum & Yes & Cecal mass, HIV+ & No recurrence, 6 mo \\
5 & $36 / \mathrm{M}$ & Sigmoid colon & No & Diarrhea, bowel obstruction, bleeding per & No recurrence, 3 yr \\
& & & rectum, HIV + & \\
\hline
\end{tabular}



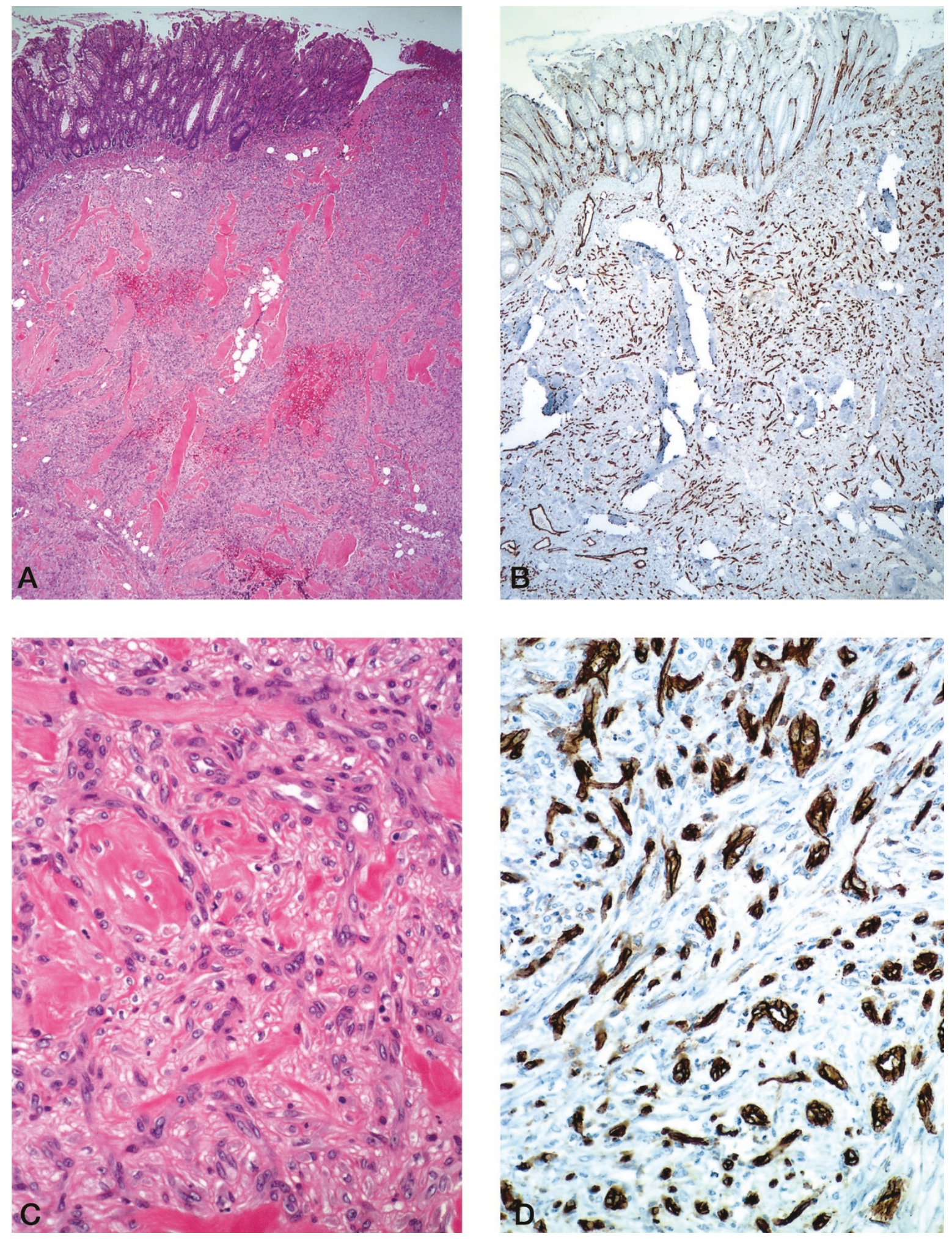

FIGURE 1. Florid vascular proliferation adjacent to chronic ulcer and extending deeply into the submucosa (A). Immunohistochemical stain for CD31 accentuates the lobular low-power architecture (B). Small vascular channels lined by bland endothelial cells (C) show strong expression of CD31 (D; Case 3).

pyogenic granuloma occurring in the intestines (17). Although our cases shared similar histologic features, they lacked the polypoid gross configuration of these lesions.
Histologic changes typically associated with intussusception include mucosal ulceration, disorganization of the muscularis propria, fusion of the muscularis mucosae with the muscularis propria, 


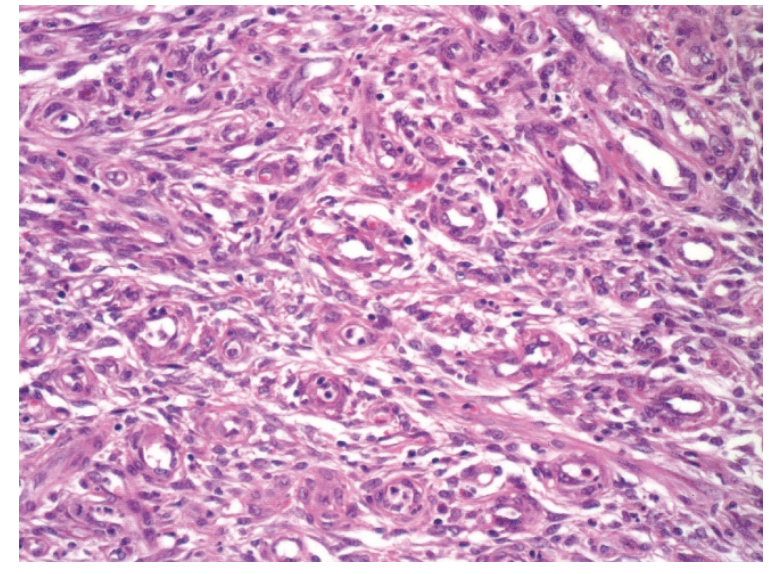

FIGURE 2. Focal area of increased cellularity and spindled cells (Case 1).

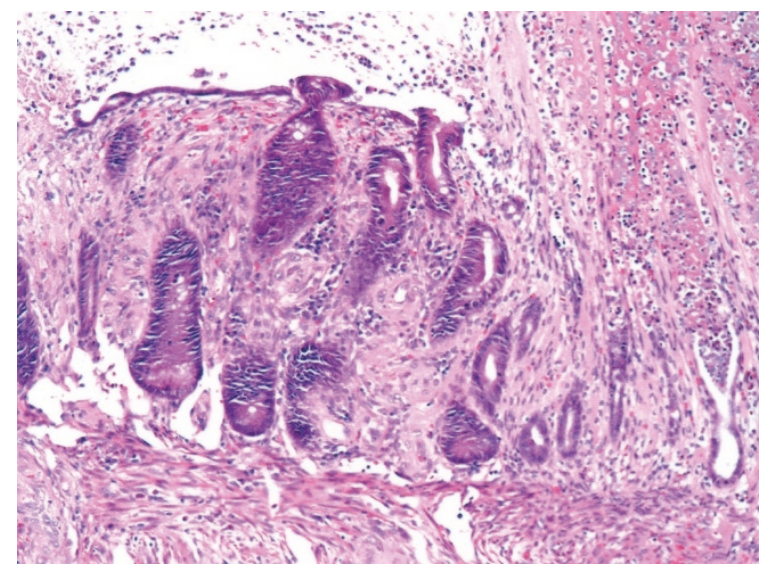

FIGURE 3. Ischemic-type changes in the mucosa adjacent to one of the lesions (Case 5).

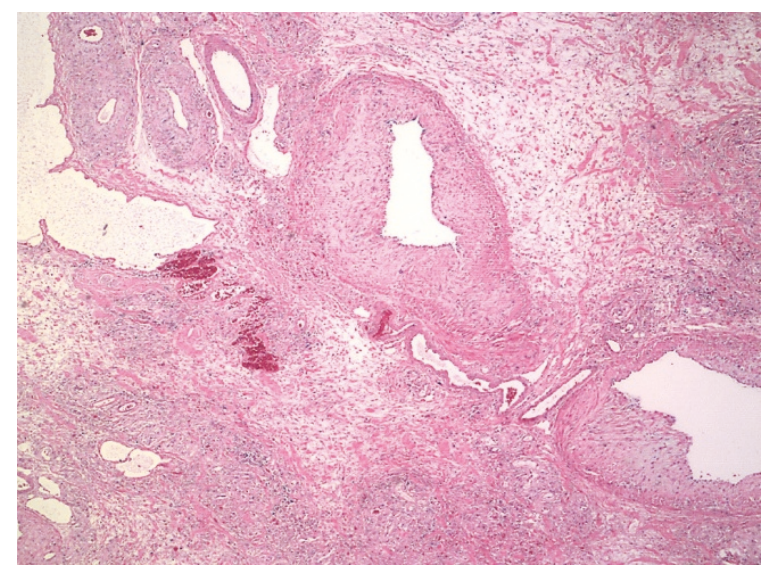

FIGURE 4. Possible arteriovenous malformation in the submucosa near one of the lesions (Case 5).

submucosal fibrosis, telangiectasia, neural hyperplasia, fibrous serosal adhesions, and mucosal hyperplasia (18). It is uncertain whether the vascular lesions we describe preceded the development of intussusception and served as a lead point or whether they resulted from longstanding mucosal prolapse associated with intussusception. It is possible that repeated mechanical forces applied to the bowel wall during prolapse or intussusception may somehow trigger angiogenesis in addition to other reactive changes. However, this hypothesis is difficult to prove. It is also possible that this process is related to the effects of repeated mechanical stress applied to a chronic ulcer, resulting in an exuberant form of highly vascularized granulation tissue.

Intussusception has been reported in adult patients with the acquired immunodeficiency syndrome, similar to two of the cases described in this report (19-27). Apparent etiologies in such cases include lymphoma $(21,23,24,27)$, mucosal lymphoid hyperplasia (26), mesenteric lymphadenopathy (28), enteric infection $(20,27,28)$, and in one case, Kaposi's sarcoma (25). The relationship between HIV infection and the lesions we observed remains obscure.

In conclusion, we describe five cases of a florid vascular proliferation of the colon in which angiosarcoma was considered in the differential diagnosis. Although the pathogenesis of the lesions was unclear, there appeared to be an association with intussusception and mucosal prolapse. Consideration of this lesion may helpful in preventing the erroneous diagnosis of angiosarcoma.

\section{REFERENCES}

1. Liebman SL, Wright TL, Altman DF. Gastrointestinal Kaposi's sarcoma in patients with acquired immunodeficiency syndrome. Endoscopic and autopsy findings. Gastroenterology 1985;89:102-8.

2. Chami TN, Ratner LE, Henneberry J, Smith DP, Hill G, Katz PO. Angiosarcoma of the small intestine: a case report and literature review. Am J Gastroenterol 1994;89:797-800.

3. Rozario A, Ravi HR. Angiosarcoma of cecum: unusual presentation with intussusception. Indian J Gastroenterol 1995; 14(1):31-2.

4. Taxy JB, Battifora H. Angiosarcoma of the gastrointestinal tract. A report of three cases. Cancer 1988;62:210-6.

5. Ramsden KL, Newman J, Moran A. Florid vascular proliferation in repeated intussusception mimicking primary angiomatous lesion. J Clin Pathol 1993;46:91-2.

6. Azar T, Berger DL. Adult intussusception. Ann Surg 1997; 226(2):134-8.

7. Begos DG, Sandor A, Modlin IM. The diagnosis and management of adult intussusception. Am J Surg 1997;173:83-94.

8. Eisen LK, Cunningham JD, Aufses AH. Intussusception in adults: institutional review. J Am Coll Surg 1999;188:390-5.

9. Gayer G, Apter S, Hofman C, Nass M, Amital R, Zissin R, Hertz M. Intussusception in adults: CT diagnosis. Clin Radiol 1998;53:53-7.

10. Pang L. Intussusception revisited: Clinicopathologic analysis of 261 cases, with emphasis on pathogenesis. South Med J 1989;82:215-28.

11. Warshauer DM, Lee JKT. Adult intussusception detected at CT or MR imaging: clinical-imaging correlation. Radiology 1999;212:853-60.

12. Boulis MN, Karp S, Rubinstein MF. Intestinal hemangioma with intussusception in infancy. J N J Med 1977;74:775-6.

13. Bower RJ, Kiesewetter WB. Colo-colic intussusception due to a hemangioma. J Pediatr Surg 1977;12:777-8. 
14. Gandhi RK, Unwadia TE, Suman GK, Shah DD. Intussuscepting haemangio-lymphangioma of the intestine in a child. J Postgrad Med 1968;14(3):136-8.

15. Gupta RK, Chopra P, Kapur BML. Intussuscepting hemangioma of the ileum in an adult. Int Surg 1970;54(4):283-5.

16. Singh RP, Sinha SN, Sinha RS. Adult jejunal intussusception secondary to angiomatous malformation. J Indian Med Assoc 1984;82(4):122-4.

17. Yao T, Nagai E, Utsunomiya T, Tsuneyoshi M. An intestinal counterpart of pyogenic granuloma of the skin: a newly proposed entity. Am J Surg Pathol 1995;19:1054-60.

18. Fenoglio-Prieser CM, Noffsinger AF, Stemmermann GN, Lantz PE, Listrom MB, Rilke FO. Pathology of the gastrointestinal tract: an atlas and text. Philadelphia: LippincottRaven: 1999.

19. Balthazar EJ, Reich CB, Pachter HL. The significance of small bowel intussusception in acquired immune deficiency syndrome. Am J Gastroenterol 1986;81:1073-5.

20. Cappell MS, Hassan T, Rosenthal S, Marcarenhas M. Gastrointestinal obstruction due to Mycobacterium avium intracellulare associated with the acquired immunodeficiency syndrome. Am J Gastroenterol 1992;87:1823-7.

21. Danin JC, McCarty M, Coker R. Case report: lymphoma causing small bowel intussusception in a patient with the acquired immune deficiency syndrome. Clin Radiol 1992;46: $350-1$.

22. Fanney DL, Silverman PM, Stull MA. Abdominal case of the day. Ileocolic intussusception in AIDS. Am J Roentgenol 1990;154:1325-6.

23. Finkle HI. Small bowel intussusception caused by lymphomatous polyposis in a patient with AIDS. Am J Gastoenterol 1997;92:726-7.

24. Gerst PH, Niazi M, Narasimha V, Saraiya R, Albu E. Primary non-Hodkin lymphoma presenting as ileocolic intussusception. Lymphology 1996;29(4):166-9.

25. Hofstetter SR, Stollman N. Adult intussusception in association with the acquired immune deficiency syndrome and intestinal Kaposi's sarcoma. Am J Gastroenterol 1988;83: 1304-5.

26. Meyerson S, Desai TK, Polidori G, Raval MF, Ehrinpreis MN. A case of intussuscpetion and lymphoid hyperplasia in a patient with AIDS. Am J Gastroenterol 1993;88:303-6.

27. Visvanathan R, Nichols TT, Reznek RH. Acquired immune deficiency syndrome-related intussusception in adults. Br J Surg 1997;84:1539-40.

28. Wood BJ, Kumar PN, Cooper C, Silverman PM, Zeman RK. AIDS-associated intussusception in young adults. J Clin Gastroenterol 1995;21:158-62. 